\title{
PRESENÇA DE PESTICIDAS EM VINHOS - UMA BREVE PROSPECÇÃO BIBLIOGRÁFICA
}

Iuri Oliveira Barbosa 1'; Lilian Lefol Nani Guarieiro 2²; Jailson B. de Andrade $3^{2 \text {; }}$ Jeancarlo Pereira dos Anjos $4^{2}$

${ }^{1}$ SENAI CIMATEC; Salvador/Bahia; iuriobarbosa@hotmail.com

${ }^{2}$ SENAI CIMATEC; Salvador/Bahia

\section{Resumo:}

O vinho tem ganhado cada vez mais popularidade entre a população, o que tem promovido um aumento na produção de uvas no mundo. Associado a isto, o uso de pesticidas nas parreiras tem crescido consideravelmente, para combater pragas nas lavouras,o que pode trazer prejuízos ao meio ambiente e seres humanos. O objetivo deste trabalho foi realizar uma breve prospecção bibliográfica sobre a presença de pesticidas em vinhos e avaliar quais países são mais relevantes no estudo deste tema, bem como a participação do. Foi realizada uma busca por artigos publicados a partir de 2010, em bases de dados como Scielo e Science Direct. Constatou-se que a China lidera no número de artigos publicados sobre a presença de pesticidas em vinhos. O estudo sobre esta temática visa a redução da contaminação desta bebida por resíduos destas substâncias, bem como contribuir para a regulamentação dos níveis de pesticidas em vinhos.

Palavras-Chaves: Vinho; pesticidas; multirresíduos

\section{PRESENCE OF PESTICIDES IN WINE - A BRIEF BIBLIOGRAPHIC PROSPECTION}

\begin{abstract}
:
Wine has gained increasing popularity among the population, which has promoted an increase in grape production in world. Associated with this, the use of pesticides in grapevines has grown considerably to combat pests on crops, which can cause damage to the environment and humans. The objective of this work was to conduct a brief bibliographic survey on the presence of pesticides in wines and to evaluate which countries are most relevant in the study of this theme, as well as the participation of Brazil. We performed a search for articles published from 2010 on databases such as Scielo and Science Direct. We have observed that China to lead in the number of articles published on the presence of pesticides in wines. The study about this theme aims to reduce the contamination of the wine by residues of these substances, as well as contribute to the regulation of pesticide levels in the beverage.
\end{abstract}

Keywords: Wine; pesticides; mutiresidues 


\section{INTRODUÇÃO}

O vinho, produto da reação da fermentação alcoólica a partir do suco de uvas logo após serem colhidas, é uma bebida apreciada mundialmente. Os tipos de vinho se diferenciam em três: suaves, espumantes e fortificados, em que a produção de cada um destes é determinada pelo tipo da uva, condições do solo, clima e processos. Esta bebida é apreciada mundialmente e movimenta diversas economias. Os Estados Unidos são os maiores consumidores, seguidos pela França e Itália. Na América do Sul, a Argentina lidera a lista de maior consumo desta bebida [1].

Como todo produto agrícola, o processo de plantio e colheita das uvas está exposto a intempéries que podem prejudicar o rendimento de colheita da lavoura. No mundo, as pragas na agricultura geram perdas de aproximadamente um bilhão de toneladas, o que é, em média, $25 \%$ do plantio. Para evitar prejuízos na colheita, a aplicação de pesticidas nas lavouras de uvas tem sido frequentemente empregada na agricultura moderna. Porém, a utilização destes gera contaminação do solo, ar, alimentos e água. Logo, a determinação quantitativa e qualitativa destes resíduos em uvas e em produtos provenientes desta fruta é importante devido à influencia na saúde humana e ecossistema que o cerca [2].

Dentre os pesticidas utilizados em lavouras de uvas, o azoxystrobin é um composto que tem sido muito utilizado. Outro pesticida potencialmente tóxico e muito utilizado no combate de pragas em lavouras de uvas é o clorpirifós. Porém, são compostos considerados tóxicos para o ambiente e à saúde humana. Muitos pesticidas são aplicados diretamente nas folhas das parreiras buscando a prevenção contra diversas doenças nas uvas, tais como a antracnose, oídio, mofo cinzento, mancha das folhas e podridão da uva madura nas videiras Após a aplicação parte destes pesticidas são incorporados a uva e se mantém dessa forma em todo o processo de produção do vinho até o seu produto final. [3].

No intuito de analisar quantitativamente a presença de pesticidas em vinhos, técnicas analíticas têm sido aplicadas durante o processo fermentativo da bebida e após seu envase. A cromatografia em fase gasosa acoplada a espectrometria de massas (CG-MS) tem sido o método mais utilizado nestas determinações [3].

O objetivo deste trabalho foi realizar uma breve prospecção bibliográfica sobre a presença de pesticidas em e avaliar quais países são mais relevantes no estudo deste tema, bem como a participação do Brasil.

\section{METODOLOGIA}

Para o levantamento bibliográfico de dados quanto a importância e relevância do tema a ser estudado, foi feita uma revisão sistemática em duas diferentes fontes de pesquisa. Assim, foram pesquisados artigos científicos nas bases de dados 
Scielo e Science Direct, cujo tema principal foi a presença de pesticidas em vinho. As buscas foram feitas utilizando três palavras-chave: wine, pesticide e analytic.

Após as buscas, com os parâmetros anteriormente mencionados, refinou-se a pesquisa por meio da seleção de artigos que continham em suas palavras-chave os três termos utilizados nas buscas (wine, pesticide $e$ analytic).

Encontrados os artigos estes foram classificados a partir do ano em que foi publicado, classificação da revista e relevância da metodologia para o tema em questão.

A pesquisa foi feita em publicações a partir de 2010 até o presente momento.

\section{RESULTADOS E DISCUSSÃO}

As buscas na plataforma Scielo apresentaram um resultado bastante reduzido para artigos com a temática sobre a presença de pesticidas em vinhos. Ao pesquisar artigos com a palavra pesticide encontraram-se 1153 artigos e, adicionando wine, 0 resultado foi reduzido a 3 publicações apenas sendo apenas uma internacional. 0 período de publicação para ambas as plataformas foi de 2010 até o presente momento (2019).

Ao realizar as buscas através do Science Direct o número de artigos relacionados ao tema pesquisado mostrou-se mais abrangente e significativo.

Utilizando a palavra de busca pesticide encontraram-se 90107 artigos, os quais se distribuíram em cada ano de acordo com a Figura 2.

Figura 1. Número de artigos por ano acerca do tema pesticidas.

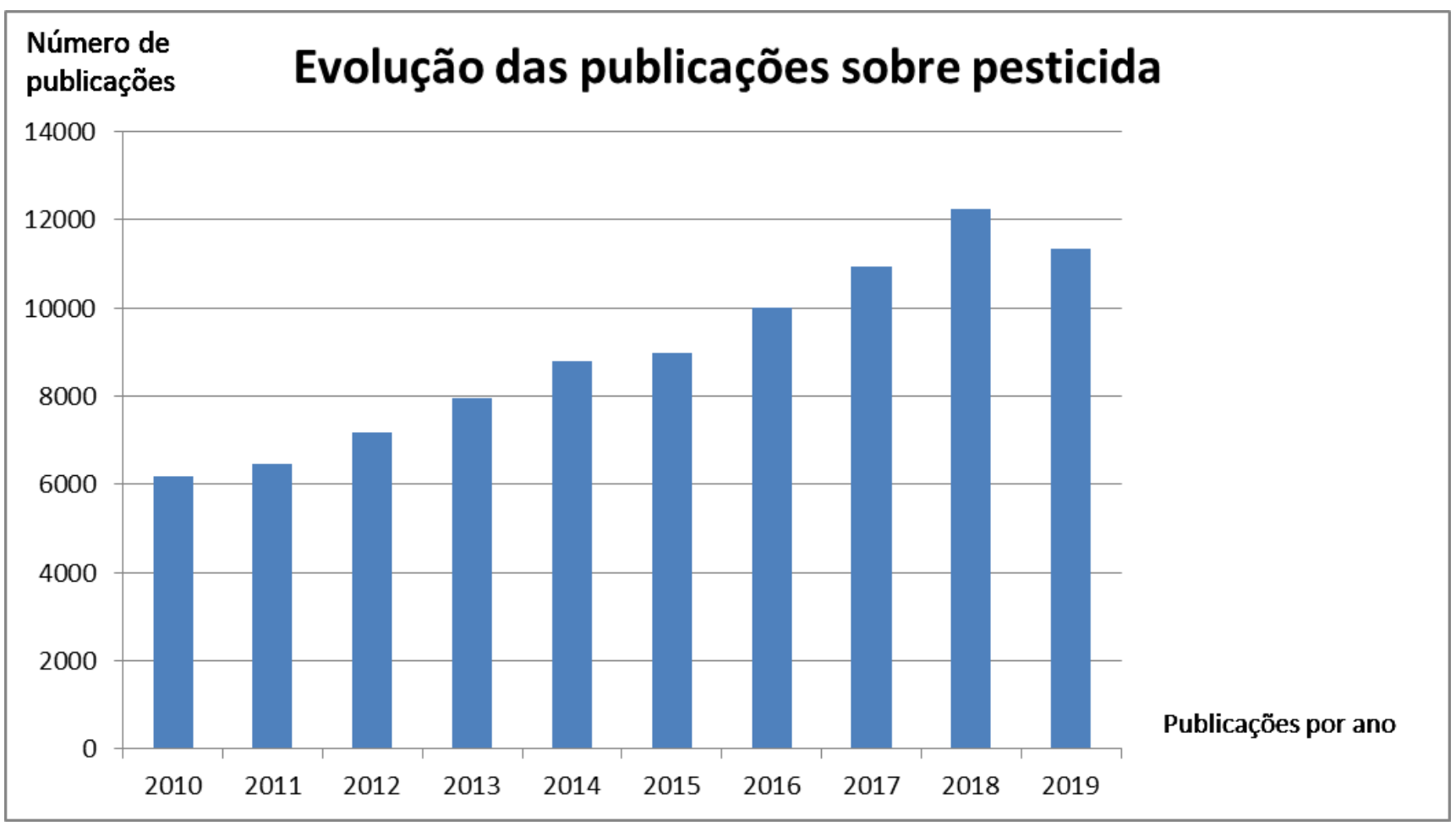


Por meio dos dados obtidos, constatou-se uma crescente busca pela pesquisa científica sobre o tema nos últimos anos. Como a pesquisa foi feita no início do mês de Agosto de 2019 acredita-se que o valor que já ultrapassa as publicações no ano de 2017 seja também superior ao quantitativo do ano de 2018.

Refinando-se ainda mais a busca adicionando a palavra wine, na busca na base de dados Science Direct, foram encontrados 4321 artigos. A fim de encontrar artigos mais específicos sobre a presença e análise de pesticidas em vinhos, e acrescentando na busca o termo analytic, foi observado um número de 2309 publicações no período pesquisado.

Adicionando ainda os três termos, simultaneamente, utilizados como busca entre as palavras chave dos artigos desejados, restringiu-se para 15 o resultado da busca mais criteriosa. Estes últimos foram objeto de uma análise mais profunda e criteriosa de conteúdo.

Levantou-se então quais os países que mais fizeram estudos acerca do tema durante o período pré-estabelecido (Tabela 1).

Tabela 1. Países com publicações mais relevantes sobre o a presença de pesticidas em vinhos.

\begin{tabular}{|c|c|}
\hline País & Quantitativo de publicações \\
\hline China & 5 \\
\hline Eslovênia & 2 \\
\hline Espanha & 2 \\
\hline Grécia & 2 \\
\hline Turquia & 1 \\
\hline Brasil & 1 \\
\hline Itália & 1 \\
\hline Bélgica & 1 \\
\hline
\end{tabular}

A China é o país que lidera a lista e nota-se uma boa prevalência de países da comunidade europeia. Vale ressaltar que a Europa lidera o ranking dos produtores de vinho tendo 5 países entre os 10 maiores produtores do mundo. França, Itália e Espanha se destacam na produção [4].

O Brasil apresenta uma publicação neste período por enquanto. Como as pesquisas sobre o tema tendem ao crescimento, nos últimos anos, espera-se um 


\section{\begin{tabular}{l|l} 
CIRCULAR ECONOMY & ECONOMIA CIRCULAR
\end{tabular}}

aumento nas publicações dos países mencionados, além de pesquisadores de outras regiões se interessarem pelo tema.

A China se destaca neste ramo de pesquisa, pois a partir de 2018 se tornou a maior produtora, consumidora e exportadora mundiais de pesticidas. Fato este que justifica a liderança deste país nas pesquisas acerca da utilização de agrotóxicos em uvas e vinhos [5].

Em seguida, analisaram-se os artigos de acordo com seu conteúdo, categoria da revista publicada, relevância do conteúdo para o tema, tipo de amostra utilizada e o ano de publicação, a fim de categorizá-los quanto a relevância do tema central da pesquisa sobre a presença de pesticidas em vinhos (Tabela 2). Notas entre 1 e 5 foram atribuídas a cada um destes quesitos.

Tabela 2. Avaliação da relevância dos artigos analisados.

\begin{tabular}{|c|c|c|c|c|c|}
\hline $\begin{array}{l}\text { Numeração } \\
\text { do artigo }\end{array}$ & $\begin{array}{l}\text { Categoria } \\
\text { da revista } \\
\text { publicada }\end{array}$ & $\begin{array}{l}\text { Relevância do } \\
\text { conteúdo para } \\
\text { o tema }\end{array}$ & $\begin{array}{l}\text { Ano em } \\
\text { que foi } \\
\text { publicado }\end{array}$ & $\begin{array}{l}\text { Tipo de } \\
\text { amostra } \\
\text { utilizada }\end{array}$ & $\begin{array}{l}\text { Pontuação } \\
\text { total }\end{array}$ \\
\hline 4 & 5 & 5 & 2 & 5 & 17 \\
\hline 11 & 5 & 3 & 5 & 3 & 16 \\
\hline 12 & 5 & 4 & 3 & 4 & 16 \\
\hline 1 & 3 & 4 & 4 & 4 & 15 \\
\hline 5 & 4 & 3 & 5 & 3 & 15 \\
\hline 14 & 5 & 4 & 3 & 3 & 15 \\
\hline 2 & 5 & 3 & 2 & 4 & 14 \\
\hline 3 & 1 & 5 & 4 & 5 & 14 \\
\hline 6 & 5 & 3 & 2 & 4 & 14 \\
\hline 7 & 5 & 2 & 3 & 3 & 13 \\
\hline 10 & 4 & 1 & 4 & 4 & 13 \\
\hline 9 & 5 & 2 & 3 & 2 & 12 \\
\hline 8 & 5 & 2 & 1 & 1 & 9 \\
\hline 13 & 1 & 2 & 2 & 2 & 6 \\
\hline 15 & 1 & 2 & 2 & 2 & 6 \\
\hline
\end{tabular}


Com estes dados foi possível construir a Tabela $3 \mathrm{com}$ a quantidade de artigos encontrados e suas respectivas notas.

Tabela 3. Quantidade de artigos x notas atribuídas

\section{Quantidade}

1

2

1

3

2

1

1

2
Nota

17

16

15

14

13

12

9

6

A partir das notas atribuídas aos artigos selecionados, as publicações mais relevantes foram consideradas para uma pesquisa mais aprofundada sobre o tema.

Através das análises feitas pôde-se destacar revistas para publicação de artigo conforme Tabela 4, a qual mostra que os artigos publicados sobre a avaliação da presença de pesticidas em vinhos ocorrem em revistas de boa qualidade. 
Tabela 4. Revistas e suas respectivas classificações

\begin{tabular}{|l|c|}
\hline \multicolumn{1}{|c|}{ Revista } & $\begin{array}{l}\text { Classificação (Qualis } \\
\text { CAPES 2016 [6]) }\end{array}$ \\
\hline Microchemical Journal & A3 \\
\hline Journal of the Brazilian Chemical Society & A2 \\
\hline Food research international & A1 \\
\hline Cadernos de saúde coletiva & B2 \\
\hline Talanta & A1 \\
\hline
\end{tabular}

\section{CONCLUSÃO}

O tema investigado se mostrou bastante relevante e com crescentes publicações em periódicos, nos últimos anos. A China se mostrou a frente de outros países na área enquanto o Brasil apresentou apenas uma publicação nos últimos 10 anos. O uso de pesticidas em diversas áreas da agricultura visa a redução da porcentagem de perdas na colheita das uvas, o que se dá principalmente devido a infestações de pragas nas parreiras. Entretanto, uma utilização indevida e excessiva destas substâncias tem potencial risco ao meio ambiente e a saúde humana, devido à contaminação por resíduos em produtos produzidos a partir de uvas contaminadas. Assim, torna-se importante uma investigação mais detalhada e aprofundada sobre a presença de pesticidas em vinhos, visando a redução da contaminação desta bebida por resíduos destas substâncias, bem como contribuir para a regulamentação dos níveis destes compostos na bebida.

\section{Agradecimentos}

Agradeço ao SENAI CIMATEC por toda estrutura disponibilizada para o desenvolvimento desta prospecção bibliográfica.

\section{REFERÊNCIAS}

1. BERTAGNOLLI, Silvana Maria Michelin et al . Natural sparkling guava wine: volatile and physicochemical characterization. Cienc. Rural, Santa Maria , v. 47, n. 9, e20151509, 2017. 
2. GALLINARI, G. SECRETARIA DE AGRICULTURA FAMILIAR E CORPORATIVISMO 3. ed. rev. amp. São Paulo: Atlas, 2019;

3. ANJOS, J,P. ANDRADE, J, B. Simultaneous determination of pesticide multiresidues in white wine and rosé wine by SDME/GC-MS. Microchemical Journal 120 (2015) 69-76

4. M. Carolina Rodríguez-Donate, Margarita E. Romero-Rodríguez, Víctor J. CanoFernández, Ginés Guirao-Pérez, Analysis of heterogeneity in the preferences of wine consumption, Wine Economics and Policy, Volume 8, Issue 1, 2019, Pages 69-80.

5. Hua Li, Hua Wang, Huanmei Li, Steve Goodman, Paul van der Lee, Zhimin Xu, Alessio Fortunato, Ping Yang,The worlds of wine: Old, new and ancient, Wine Economics and Policy, Volume 7, Issue 2, 2018, Pages 178-182.

6. Qualis novo. Plataforma Sucupira. Disponível em:

https://sucupira.capes.gov.br/sucupira/public/consultas/coleta/veiculoPublicacaoQual is/listaConsultaGeralPeriodicos.jsf. Acesso em 01/08/2019. 